between time taken to make risky $(t(28)=-1.28, \mathrm{p}=\mathrm{ns})$ and non-risky choices $(t(28)=-1.06, \mathrm{p}=\mathrm{ns})$. Similarly, no difference was found for change in risky choice selection in presence of the drug $(t(28)=-1.41, \mathrm{p}=\mathrm{ns})$. No differences were found in the traditional reinforcement learning parameters between the groups.

Conclusions Using a novel computational analysis, we showed that dopaminergic medication increased the preference to select a risky choice by modulating drift rate and response bias which was not captured by the behavioural measures. Critically we observe an effect on response bias highlighting the role of apriori information in influencing risky decision making.

\section{LIFESPAN OF NEGATIVE EXPERIENCES IN FUNCTIONAL NEUROLOGICAL DISORDER PATIENTS}

${ }^{1}$ Aneesa Mehmood*, ${ }^{2}$ Liat Levita, ${ }^{3}$ Markus Reuber, ${ }^{2}$ Emily Mayberry. ${ }^{1}$ Sheffield Medical School; ${ }^{2}$ University of Sheffield Psychology Department; ${ }^{3}$ University of Sheffield Academic Neurology Unit

\subsection{6/jnnp-2019-BNPA.44}

Aims Exploration of the relationship between negative life experiences and patients with Functional Neurological Disorder (FND), by analysing patient and non-clinical group responses to a new questionnaire called the Lifespan of Negative Experiences Scale (LiNES). LiNES was designed to examine predisposing vulnerabilities and perpetuating factors in individuals with FND by retrospectively assessing experiences of interpersonal trauma, affect and relationship insecurity at three developmental stages - childhood, adolescence and adulthood.

Methods LiNES, CATS (measure of childhood abuse and trauma), RSQ (measure of relationship insecurity) and PANAS (measure of affect) questionnaires were administrated to 71 individuals with FND. Analyses were conducted to assess the reliability of the LiNES, explore correlations between different psychological domains within the FND group and to test whether LiNES scores predicted FND group membership. In addition, FND patients' responses where compared to 270 matched healthy controls.

Results The LiNES subscales had high internal consistency and correlated with CATS, RSQ and PANAS. Levels of interpersonal trauma were higher in FND patients than controls during childhood, adolescence and adulthood. High levels of negative affect were found in FND patients in adulthood compared to controls but no significant differences were found between FND patients and controls in relationship insecurity at any developmental stage. On the RSQ, FND patients had higher anxious and avoidant relationship styles. LiNES trauma scores at each developmental stage predicted FND status with over $80 \%$ accuracy. Additionally, FND patients self-reported more symptoms (SDQ-20) and a higher prevalence of comorbid conditions compared to controls.

Conclusions The LiNES is a new brief retrospective measure of negative life experiences. Although psychological factors may not be necessary to the diagnosis of FND, they are substantially more common in FND patients compared to controls. In particular, a history of interpersonal trauma seems to play an important role in those with FND. These factors therefore are likely to play a pathophysiological role in many patients and their recognition is important for treatment. This study provides new insights into the association between the timing of negative experiences and the subsequent effect on an individual. Furthermore, the results support the use of LiNES as a valid screening tool in the clinical setting in patients presenting with functional symptoms with diagnostic and therapeutic implications.

\section{FUNCTIONAL NEUROLOGICAL DISORDER IN GERIATRIC REHABILITATION: INCIDENCE, CLINICAL PRESENTATIONS, AND IMPACT ON DISCHARGE}

${ }^{1}$ Corretge Maria, ${ }^{1,2}$ Chun Ho-Yan Yvonne, ${ }^{1}$ Roscoe Mhairi, ${ }^{2}$ Carson Alan. ${ }^{1}$ Department of Medicine of the Elderly, St John's Hospital, Livingston, UK; ${ }^{2}$ Centre for Clinical Brain Sciences, University of Edinburgh

\subsection{6/jnnp-2019-BNPA.45}

Background/Aims Functional neurological disorder (FND) may be present amongst elderly people in hospital. FND could hinder patients' rehabilitation progress and impact negatively on discharge outcomes. Little data exist for FND in the elderly. We aimed to report the incidence of FND, clinical presentations, co-morbidities, and impact of FND on discharge in elderly patients receiving inpatient rehabilitation.

Methods In our retrospective case series, a consultant geriatrician reviewed electronic case notes of consecutive discharges from a 28-bed geriatric rehabilitation unit at St John's Hospital, which serves all patients requiring inpatient rehabilitation in West Lothian - a mixed rural and urban area with a population of 180000 and high levels of deprivation. Data collected: demographics, suspected/definite diagnosis of FND and its presentation, significant co-morbidities and impact on discharge.

Results We reviewed case notes of 100 patients discharged consecutively from 30/3/2018 to 30/10/2018 (age range 41-101, mean 79 , SD $11 ; 55 \%$ men). $20 \%$ received a diagnosis of suspected or definite FND. FND diagnosis was made by a geriatrician (17\%) or a neurologist (3\%). Clinical description of FND cases and their co-morbidities will be presented in a summary table. Of the 20 FND cases (mean age 77, SD 14), 9/20 (45\%) were men. FND impacted on discharges in 13/20 (8/20 had delayed discharge, 5/20 had increased care needs, $7 / 20$ had no impact on discharge).

\section{Conclusion}

Key finding FND was common amongst elderly patients receiving inpatient rehabilitation. FND presentations were varied. Patients with FND also had chronic conditions common in the elderly e.g. Parkinson's disease, stroke, dementia, anxiety or depression.

Weakness and strength of our study Assessor bias might be introduced as diagnosis was made by a geriatrician with an interest in neuropsychiatry. Our data are likely generalisable to the geriatric rehabilitation population as sample was obtained from the only unit that served the entire population of West Lothian.

Implications for future research and practice FND presents a unique challenge in the geriatric population. Geriatricians are not accustomed to assessing and managing FND, sometimes dismissing symptoms as 'behavioural'. This can lead to symptoms remaining unexplained and untreated. Specialist neurology or neuropsychiatry services are not always available.

The identification of FND and its effective treatment during rehabilitation could have potential impact on hospital length of 